\title{
RECENT ADVANCES IN MEASUREMENT TECHNIQUE OF MICROSCALE THERMOGRAPHY
}

\author{
Junko Morikawa \\ Tokyo Institute of Technology, School of Materials science and Chemical Technology, Tokyo 152- \\ 8550, Japan \\ morikawa.j.aa@m.titech.ac.jp
}

\begin{abstract}
In order to analyze the recent problems in thermal management, micro-scale thermography has been utilized to measure the thermophysical properties of energy materials and to detect the localized failure in semiconductors in the electronic industrial parts. Our equipment is widely applicable in the field of microscale thermography to achieve high-quality thermal imaging. The Infrared (IR) optical lens design has been optimized to each wavelength band of the photon type and the thermal type detectors of IR FPA. Typical applications to observe the freezing biological cells and the crystallization of organic mlecular crystals are reviewed together with the microscale laser flying spot method using the superimpose technique. The recent instrumentation of thermospectroscopy and the high temperature imaging systems are introduced with regard to the exothermic heat of chemical reaction in polymerization in microfluidics and the chemical heat storage process with molten salt to be utilized as the energy carier for the solar power plant.
\end{abstract}

KEYWORDS: microscale thermography, temperature wave, IR micro lens, freezing, crystallization, flying spot, superimpose, microfluidics, , thermospectroscopy, molten salt, chemical heat storage

\section{INTRODUCTION}

The concept of instrumentation of the analytical instrument for quantitative microscale thermography is aimed at two directions; active thermography, and visual thermal analysis that can be applied to both a photon type and a thermal type IR detector arrays. In this study, originally developed two algorithms are examined, 1. Pixel by pixel emissivity calibration, and 2. Pseudo acceleration. From the viewpoint of general use in industry, the instrumentation with thermal IR detector arrays is available to realize the portable size analytical instrument.

The photon detectors are superior in speed and sensitivity in thermal and long IR wavelength (LWIR), and micro-scale thermography has been usually operated with InSb detectors combined with the optical lens design optimized for $3 \mu \mathrm{m}$ to 5 $\mu \mathrm{m}$ wavelengths. [1-2] At the same time, the method has been utilized as a thermal microscope to observe the crystallization of organic materials and to detect an invisible failure and defects in semiconductors or microstructures of composite materials by an input of pulsed or modulated light sources. [3] These are called as the micro-scale thermography. [4] However, in order to achieve a portable instrumentation for a practical use in industry with a smaller amount of specimens, an un-cooled micro-bolometer (thermal detector) is chosen as an IR-FPA sensor combined with the superimpose technique including the above mentioned two algorithms. [5]

Based on these technical backgrounds, the additional new instrumentation systems are developed regarding the thermospectroscopy and the high temperature imaging systems as for the topics as follows:

(1) chemical reaction in styrene radical polymerization in microfluidics activated on the interface of the co-flows of AIBN toluene solution and pure styrene monomer (at $\left.20-80^{\circ} \mathrm{C}\right),[6]$

(2) in the solidification from the molten flows of the solar salts, sodium nitrate, and potassium nitrate in the exothermic heat front, $\left(200-350^{\circ} \mathrm{C}\right)[7]$

(3) the carbonation and decarbonation process in a lithium orthosilicate/carbon dioxide $\left(\mathrm{Li}_{4} \mathrm{SiO}_{4} / \mathrm{CO}_{2}\right)$ reaction of chemical heat storage process (at $\left.600-800{ }^{\circ} \mathrm{C}\right)$. [8] 


\section{INSTRUMENTATION}

\subsection{THEERMO-SPECTROSCOPY}

The instrument is composed of a grating monochromator to select particular wavelengths of infrared light (CT-10, JASCO Corporation), a ceramic heater, a chopper, a condenser module with a parabolic mirror, a thin hot stage (S84, ST Japan) and the IR camera (Phoenix, Indigo). The monochromator used in this study is a typical wavelength dispersion type that is composed of 2 slits and diffraction grating. A blazed grating with 300 grooves was used to scan over a broad infrared band ranging from 2.76 $\mu \mathrm{m}$ to $5.24 \mu \mathrm{m}$ in $0.4 \mathrm{~nm}$ steps in this application $\left(3623 \mathrm{~cm}^{-1}\right.$ to $\left.1908 \mathrm{~cm}^{-1}\right)$ with a wavelength resolution of $33 \mathrm{~nm}$ or $3.29 \mathrm{~cm}^{-1}$. The IR light emitted from the slit is monochromatic and condensed with a parabolic mirror of $1 / 3$ reduction rate. The IR camera is an InSb focal plane array (FPA) composed of $320 \times 256$ pixels and pitch size of $30 \mu \mathrm{m} \times 30 \mu \mathrm{m}$ with $\times 7$ magnification objective lens, the spatial resolution is $4 \mu \mathrm{m}$.

The thermal and the spectroscopic images are simultaneously recorded by synchronizing signal of chopper and the trigger signal for frame acquisition of the camera, which attains the high simultaneity in the measurement. The simultaneous acquisition of thermal and spectroscopic images was performed at $200 \mathrm{~Hz}$, alternating acquisition of two kinds of images, one is the thermal + monochromatic infrared light ( $S$ image) and the other is the thermal ( $E$ image). From these two experimental image data, the monochromatic infrared transmitted light image ( $I$ image) can be obtained. By changing the wavelength of the incident monochromatic light continuously, the $I$ image at each wavelength is measured and spectra at each pixel can be measured. The relation between these three images can be described as $S=E+I$.

\subsection{THERMAL IMAGING IN HIGH TEMPERATURES}

For the purpose of high temperature measurements up to $900{ }^{\circ} \mathrm{C}$, the specified thermal insulation design was required for the heating hot stage, a sample holder and the optics lens system to avoid the convection and radiation from the surrounding high temperature. A concentrated plane coil micro-heater of diameter $8 \mathrm{~mm}$, power of $50 \mathrm{~W}$, was embedded in the $8 \mathrm{~mm}$ diameter Mullite tube pipe sintered with alumina cement in the heating stage. The high temperature was insulated by the double thermal shading structures with the inert gas in order to avoid the corrosion. The working distance of a retrofocus type objective lens adjusted to the IR waveband was designed longer than $25 \mathrm{~mm}$ to minimize the influence from the thermal radiation through the vessel. The micro-bolometer VOx IR FPA (FLIR Tau2) was chosen as the FPA sensor, with $17 \mu \mathrm{m}$ pixel pitch, 640 x 512 pixel numbers, with $60 \mathrm{~Hz}$ frame rate, with 8-12 $\mu \mathrm{m}$ spectral waveband.

The efficient applicability of microscale thermography to various kinds of thermal management will be shown in the presentation.

\section{ACKNOWLEDGMENT}

This work was supported in part by Council of Science, Technology and Innovation (CSTI), Cross-ministerial Strategic Innovation Program (SIP), "Energy Carrier" (Foundation agency JST) and by a JSPS KAKENHI Grant No. 16K06768.

\section{REFERENCES}

[1] T. Hashimoto, J. Morikawa, " Two-dimensional thermal analysis on freezing of onion epidermal cell by high-speed infrared microscopic camera," Jpn. J. Appl. Phys. 42 (2003) L706-L708.

[2] C. Pradere, J. Morikawa, J. C. Batsale, T. Hashimoto, "Microscale thermography of freezing biological cells in view of cryopreservation," Quantitative Infra Red Thermography Journal 6 (2009) 37.

[3] J. Morikawa, E. Hayakawa, T. Hashimoto, R. Buividas, S. Juodkazis, "Thermal imaging of a heat transport in regions structured by femtosecond laser", Opt. Exp. 19(2011) 20542-20550.

[4] J. Morikawa, " Microscale IR Thermography, " 6 May 2014, SPIE Newsroom. DOI: 10.1117/2.1201405.005455.

[5] J. Morikawa, E. Hayakawa, T. Hashimoto, " Two-dimensional Thermal Analysis of Organic and Polymeric Materials with cooled and uncooled infrared cameras", Advances in Optical Technologies 2012 (2012) 484650.

[6] M. Ryu, J. A. Kimber, T. Sato, R. Nakatani, T. Hayakawa, M. Romano, C. Pradere, A. A. Hovhannisyan, S. G. Kazarian, J. Morikawa, Infrared thermo-spectroscopic imaging of styrene radical polymerization in microfluidics, Chem. Eng. J. 324 (2017) 259-265.

[7] J. Morikawa, M. Zamengo, Y. Kato, Superimpose signal processing method for micro-scale thermal imaing of solar salts at high temperatures, Proc. SPIE 9861, p. 98610L, 2016.

[8] J. Morikawa, H. Takasu, M. Zamengo, Y. Kato, Micro-scale thermal imaging of CO2 absorption in the thermochemical energy storage of Li metal oxides at high temperature, Proc. SPIE 10214, p. 1021408, 2017. 